\title{
Premature Ovarian Insufficiency: An Important Yet Neglected Health Condition
}

\author{
Syeda Batool Mazhar ${ }^{1,2}$, Zahra Muslim ${ }^{3}$ \\ ${ }^{1}$ Professor \& Head Department of Obstetrics \& Gynecology, MCH Centre, PIMS, Islamabad Pakistan \\ ${ }^{2}$ Pro Vice Chancellor, Shaheed Zulfiqar Ali Bhutto Medical University, Islamabad Pakistan \\ ${ }^{3}$ FCPS Resident, Department of Obstetrics \& Gynecology, MCH Centre, PIMS, Islamabad Pakistan
}

Menopause is defined to occur retrospectively at the completion of 12 months after the last reported menstrual period. This is due to ovarian follicular depletion along with estrogen deficiency. ${ }^{1}$ The average age at menopause is variously reported between 47 - 52 years in different populations worldwide. Almost $10 \%$ women report early or premature menopause before 45 years. Another $1 \%$ have premature ovarian insufficiency (POI) under the age of 40 while about 1 in 1000 women have POI under the age of 30 years. ${ }^{2}$ The women with POI report vasomotor symptoms like hot flushes and night sweats affecting their quality of life. Menopause at an early age has long term adverse cardiovascular outcomes associated with increasing central obesity, atherogenic lipid profile and glucose intolerance. ${ }^{3,4}$ Other health concerns in $\mathrm{POI}$ include fertility, contraception, neurological, psychological, psychosexual function and bone health. Every year, $18^{\text {th }}$ October is celebrated as World Menopause Day and the theme for the year 2020 is POI.

POI can present either as primary or secondary amenorrhea. The etiology of premature ovarian insufficiency is classified broadly as autoimmune, non immune and iatrogenic. A reliable diagnostic

Correspondence:

Syeda Batool Mazhar

Email: batoolmazhar@yahoo.com

Cite this editorial: Mazhar SB, Muslim Z. Premature Ovarian Insufficiency: An Important Yet Neglected Health Condition. J Islamabad Med Dental Coll. 2021; 10(1): 1-3

Doi: 10.35787/jimdc.v10i1.664 test for autoimmune $\mathrm{POI}$ is not available and treatment strategies for autoimmune POI lack expert consensus. Nonimmune conditions are often due to genetic mutations involved in the in-utero ovarian development sequence. This may result in delayed puberty, primary amenorrhea, or premature ovarian insufficiency (POI) as seen typically in Turner's syndrome and gonadal dysgenesis. Young cancer patients in modern oncology practice have up to $75 \%$ long term survival. Chemotherapy and or radiation therapy can affect the HPG axis at different levels related to the chemotherapeutic drug type and the field of irradiation. Alkylating agents like cyclophosphamide are most gonadotoxic with high incidence of follicular depletion in females due to destruction of oocytes. Radiation is particularly harmful for the germ cells of the gonads. Brain tumors requiring CNS irradiation often results in pituitary hormone deficiencies including gonadotropin deficiency which may occur many years post CNS irradiation entailing long term follow up in young cancer survivors. Bone marrow transplant for various hematologic disorders and inherited metabolic disorders also results in delayed puberty. Counseling the parents for fertility preservation is very important for pediatric patients with cancer. Gonadal protection from chemotherapy can be achieved to some extent by ovarian suppression with GnRh agonist injections and or radiation protection by gonadal shielding and surgical transposition of ovaries out of pelvis. Oocyte preservation is usually not feasible in majority of 
cases due to prohibitive costs and limited availability in Pakistan. While taking informed consent for any medical or surgical intervention likely to result in $\mathrm{POI}$, counseling of the patient and her family is very important.

In girls with delayed puberty, medical history for anosmia, eating disorders, chronic inflammatory bowel disease, malabsorption syndromes and hemoglobinopathies should be elicited and investigated based on clinical features. Any family history of involuntary subfertility and delayed puberty is noted. Physical examination for height, weight, BMI, breast development, axillary and pubic hair is essential. To exclude chronic disorders, inexpensive investigation like erythrocyte sedimentation rate, renal function tests and urinalysis can be used for screening. Hormone assays include serum LH, FSH, dehydroepiandrosterone sulfate (DHEAS), estradiol (E2), testosterone, prolactin, TSH and Anti-Mullerian hormone levels. Ultrasonography pelvis and abdomen remains the first line imaging modality. The bone age should be assessed with $X$ ray study of the limb bones while more advanced imaging like brain MRI remains limited to specific indications. Elevated gonadotropin concentrations suggest primary gonadal failure especially when skeletal maturation is more than 11 years. If gonadotropin concentrations are elevated, chromosomal analysis should be performed in all women with noniatrogenic POI especially for Turner's syndrome and gonadal dysgenesis. Gonadectomy is recommended for women with detectable $Y$ chromosomal material. Genetic testing for autosomes is done if history and examination indicates a specific mutation. 6 After excluding pregnancy as the cause of amenorrhea, progesterone withdrawal can be performed to assess effects of endogenous estrogen on uterus as well as patency of the genital outflow tract.
Implications for siblings or other relatives needs due consideration. Currently there is no established predictive test for $\mathrm{POI}$ or established prevention measures to offer to female relatives of $\mathrm{POI}$ women. Serum FSH ( $>25 \mathrm{mIU} / \mathrm{ml}$ ) and E2 level $(<30 \mathrm{pg} / \mathrm{ml})$ repeated 4 weeks apart are used to diagnose $P O I$ in clinical practice. Serum AMH $(<1.0 \mathrm{ng} / \mathrm{ml})$ levels are also suggestive. For puberty induction in girls with primary $\mathrm{POI}$, low dose oral estrogen in gradually increasing doses given over the next three years. Cyclical progestogens are added after minimum two years of estrogen. The oral contraceptive pill is not recommended for hormone replacement to induce puberty.

A woman's health and wellbeing are affected by her environment and cultural influences, not merely the age at menopause. The management options range from lifestyle interventions to hormonal and nonhormonal treatments with specific benefits and risks. Women at risk of $\mathrm{POI}$ should be advised to stop smoking, maintain optimal weight with a balanced diet and regular exercise of 120-150 min per week. Adequate intake of calcium and vitamin $D$ with supplementation as indicated needs to be ensured. Women with POI have about $5 \%$ chance of spontaneous pregnancy. No effective fertility interventions to increase natural conception rates are available. Anecdotal experience with DHEA 25 $\mathrm{mg}$, 8-12 hourly for 6 months is reported in literature with conflicting results. Stem cells in the form of PRP injections to ovaries as well as in vitro activation of primordial follicle for $\mathrm{POI}$ patients are being researched.7 Oocyte donation is recommended for fertility in women with POI but is not legal in Pakistan. This needs to be revisited by the national legal, medical and religious experts.

Decisions about hormone replacement therapy for POI are based on separate evidence and recommendations compared to women with age more than 45 years at menopause. 6 Early initiation of hormone replacement therapy (HRT) is 
recommended in women with POI for optimal health outcomes and it should be continued "at least until the average age of natural menopause". There is no risk of increased breast cancer associated with HRT in the women with POI. Annual monitoring of blood pressure and weight is however recommended as a good practice point.

Increasing awareness of condition of POI with optimal management can go a long way to improve quality of life of this group of women with re duction in long term morbidities and better health outcomes.

\section{References}

1. Davis SR, Lambrinoudaki I, Lumsden M, Mishra GD, Pal L, Rees M, et al. Menopause. Nat Rev Dis Primers. 2015; 1: 15004. Doi.org/10.1038/nrdp.2015.4.

2. Albright $F$, Smith $P$, Fraser R. A syndrome characterized by primary ovarian insufficiency and decreased stature. Am J Med Sci. 1942; 204: 625-48.

3. Anagnostis P, Christou K, Maria Artzouchaltzi A, Gkekas NK, Kosmidou N, Siolos P, et al. Early menopause and premature ovarian insufficiency are associated with increased risk of type 2 diabetes: a systematic review and meta-analysis. Eur J Endocrinol. 2019; 180: 41-50. Doi: 10.1530/EJE-180602.

4. Muka T, Oliver-Williams C, Kunutsor S, Laven JS, Fauser BC, Chowdhury R, et al. Association of age at onset of menopause and time since onset of menopause with cardiovascular outcomes, intermediate vascular traits, and all-cause mortality: a systematic reviewand meta-analysis. JAMA Cardiol. 2016; 1(7): 767-76. Doi: 10.1001/jamacardio.2016.2415.

5. Kirshenbaum M, Orveito R. Premature ovarian insufficiency (POI) and autoimmunity-an update appraisal. J Assist Reprod Genet. 2019; 36(11): 2207 15. Doi: 10.1007/s10815-019-01572-0.

6. Webber L, Davies M, Anderson R, Bartlett J, Braat D, Cartwright $B$, et al. ESHRE Guideline: Management of women with premature ovarian insufficiency. Hum Reprod. 2016; 31(5): 926-37. Doi: 10.1093/humrep/dew027.

7. Lee HN, Chang EM. Primordial follicle activation as new treatment for primary ovarian insufficiency. Clin Exp Reprod Med. 2019; 46(2): 43-9. Doi: 10.5653/cerm.2019.46.2.43. 